\title{
Treatment of Isotopic Exchange Reactions Having Complex Mechanisms*
}

\author{
R. A. Marcus \\ Department of Chemistry, Polytechnic Institute of Brooklyn, Brooklyn 1, Nerv York
}

(Received October 26, 1954)

\begin{abstract}
The reaction rate vs time data of isotopic exchange reactions have been shown by previous work to obey a first-order rate law, neglecting differences in isotopic reactivity. A simple method, of use in reactions having complex mechanisms, is developed for relating this first-order rate constant to the rate constants of elementary steps in the process. The method involves examining the progress of specified atoms through the reaction sequence, and is applicable regardless of the number of exchange positions of the reactants, or of whether or not one isotope is present in tracer amounts. These results are used to reinterpret some data on the deuteration of diborane.
\end{abstract}

\section{INTRODUCTION}

$\mathrm{D}$ URING the past few years there has been a considerable amount of research on the rates of isotopic exchange reactions. Neglecting differences in isotopic reactivity, simple ${ }^{1}$ calculations show that the rate at which the over-all isotopic composition of any chemical species in these reactions approaches its final value should obey a first-order rate law, regardless of their mechanism. A chemical species is defined here as a class of compounds differing only in their isotopic composition. This conclusion that the rate of formation of an equilibrium distribution of the isotopes among the reactants from an initially nonequilibrium distribution should be first order has been abundantly confirmed experimentally. The explicit form of the rate law ${ }^{1}$ is given by

$$
R t=-\frac{a b}{a+b} \ln (1-F),
$$

where $a$ and $b$ denote the concentrations of exchangeable atoms in the two reactants $A$ and $B$, in gram atoms per liter say, $R$ denotes the rate per unit volume at which atoms in $A$ pass over to positions in $B$, and $F$ denotes the fraction of exchange at time $t$. The rate per unit volume at which atoms in $B$ pass over to positions in $A$ must of course equal $R$. Furthermore, $F$ is defined as $\left(x_{a}^{0}-x_{a}\right) /\left(x_{a}{ }^{0}-x_{a}^{\infty}\right)$, where $x_{a}, x_{a}{ }^{0}, x_{a}{ }^{\infty}$ denote the fractions of exchangeable atoms in $A$ which are present as a particular isotope, $\alpha$ say, at time $t, 0$, and $\infty$.

When the mechanism of the exchange reaction is complex in that the over-all reaction is composed of several elementary steps, a problem of major interest is the relation of the quantity estimated from the experimental data, $R$, to the rate constants of the elementary steps and to the concentration of the reactants. In the present paper a simple method of obtaining this relation is presented and compared with other methods which have been, or could be used. Application of this technique to some recent data is made.

\footnotetext{
* This work was supported in part by the Office of Naval Research under Contract No. Nonr 839(09).

1 A. C. Wahl and N. A. Bonner, Radioactivity Applied to Chemistry (John Wiley and Sons, Inc., New York, 1951), p. 7, give references to the original proof of $\mathrm{H}$. McKary and to other derivations; G. M. Harris, Trans. Faraday Soc. 47, 716 (1951).
}

\section{THEORETICAL TREATMENT}

\section{General Discussion}

It is useful to recall for purposes of continuity the simple proof ${ }^{1}$ of Eq. (1). From the definitions of $x_{a}$ and $R$ it follows that the rate per unit volume in which atoms in $A$ of the recognizable isotopic type, $\alpha$ say, pass over to positions in $B$ is $R x_{a}$ and the rate at which atoms in $B$ of the same isotopic type pass over to positions in $A$ is $R x_{b}$, where $x_{b}$ is the fraction of exchangeable $B$ atoms which are of the isotopic type $\alpha$. Thus the net rate at which these atoms pass from $A$ to $B$ is

$$
-\frac{d}{d t}\left(a x_{a}\right)=R\left(x_{a}-x_{b}\right)
$$

where $x_{a}$ and $x_{b}$ are related by the material balance equation

$$
a x_{a}+b x_{b}=a x_{a}^{\infty}+b x_{b}^{\infty}=(a+b) x_{a}^{\infty},
$$

which involves the fractions at infinite time. The second part of this equation follows from the fact that at equilibrium, i.e. at $t=\infty$, the fraction of exchangeable atoms of species $A, x_{a}^{\infty}$, which are of the isotopic type $\alpha$ equals the corresponding fraction $x_{b}^{\infty}$ in the species $B$. It immediately follows from the elimination of $x_{b}$ between Eqs. (2) and (3) that

$$
-\frac{d x_{a}}{d t}=R \frac{a+b}{a b}\left(x_{a}-x_{a}^{\infty}\right) .
$$

Equation (1) then results when Eq. (4) is integrated from $t=0$ to $t=t$.

One method of obtaining a detailed interpretation of $R$, which has been used in tracer studies of reactions having complex mechanisms, is applicable to mechanisms involving only one slow step. For example, it can be applied to reaction systems having several rapidly attainable equilibria which form intermediates, followed by a slow reaction between these intermediates (or between an intermediate and a reactant). The rate $R$ is simply the rate expression for the reaction of these intermediates. It therefore involves their concentrations and these may be expressed in terms of the stoichiometric concentrations of the reactants using the appro- 
priate equilibrium constant expressions. This method is simple and extremely useful but it is nevertheless of limited applicability.

If one of the isotopes is present in tracer amounts, denoted as the labeled isotope, then another more general way of obtaining the relation between $R$ and the rate constants of the elementary steps could involve setting up expressions for the rates of formation and disappearance of each of the labeled reactants and intermediates. Once again a first-order law can be shown to result, only now the first-order constant has the desired form. It explicitly contains the rate constants of the elementary steps and is, generally, some function of the concentrations of the reactants. This method, however, becomes increasingly laborious with increasing complexity of the reaction mechanism, and a related, though appreciably simpler method of evaluating $R$ is developed in the next section.

If both isotopes are present in comparable amounts but each type of molecule present contains at most one exchangeable atom, then a somewhat similar procedure to that used for the tracer case may be employed, or the procedure discussed in the following paragraph may be used.

When the isotopes are present in comparable amounts and at the same time the reactants have several exchangeable positions, many isotopically different compounds are formed, some isotopically isomeric with the intermediates and some with the reactants. In the case of complex mechanisms this enormously complicates any attempt to solve the equations for the rate of change of concentration of each of the many isotopically distinguishable molecules present. ${ }^{2}$ For example, because of this isotopic multiplicity, it would be necessary to consider a large number of reactions, of the order of one hundred, in treating the exchange reaction of $\mathrm{B}_{2} \mathrm{H}_{6}$ with $\mathrm{D}_{2}$ to be described later. In the present paper attention will be focused on the more limited but simpler problem of interpreting in terms of a detailed mechanism the rate of change of the over-all isotopic composition of any chemical species, i.e., of any class of compounds differing only in their isotopic composition. To accomplish this, it is first observed that this rate of isotopic exchange is given by Eq. (1), for the derivation of that equation was seen to be quite general. The problem then becomes one of evaluating the constant $R$ occurring in this equation, in terms of the rate constants of the elementary steps of a suitable detailed reaction mechanism. To do this we note that $R$ is independent of isotopic composition; therefore, its value for this apparently more complicated case, where the amounts of the isotopes are comparable and the reactants have several exchangeable positions, is the same as that for the case where one of the isotopes is present in tracer amounts. That is, $R$ for the complicated case may be

${ }^{2}$ An exception to this comment may be found in initial rate studies, for initially only relatively few distinguishable molecules are present. evaluated by determining it for the tracer case. Some methods for doing this have already been mentioned while in the following section a general simple treatment, valid for both cases, will be developed.

\section{Evaluation of $R$}

\section{General}

In order to relate $R$ to the rate constants of the elementary steps of the isotopic exchange reaction between $A$ and $B$ and to the initial concentrations, the progress of certain atoms through the reaction sequence from $A$ to $B$ will be followed. This will be done by labeling a certain, very small number of atoms which are initially in the $A$-type molecules say. This number of labeled atoms is taken to be so small that the number of molecules, $A$-type or otherwise, containing two labeled atoms per molecule is negligible and that the chance of a reaction between two molecules, each containing one labeled atom, is also negligible.

Let $A^{*}$ be the concentration of those $A$-type molecules which at time $t$ have one labeled atom each. An artifice which we find simplifies considerably the detailed evaluation of $R$ will now be introduced. We will let the label* be such that by definition it vanishes when the specified atom enters a position in a $B$-type molecule, but not when the specified atom merely enters a position in one of the intermediates. Therefore $A^{*}$ is actually the number of specified atoms per unit volume which at time $t$ have never reached a position in a $B$-type molecule. It follows that $A^{*}$ will decrease steadily with time from its initial value at $t=0$ to zero at $t=\infty$. The simplification resulting from the introduction of this artifice is that reactions involving the redissociation of a $B$ molecule containing what would have been a labeled atom need not be considered for the evaluation of $R$. Detailed examples of this simplification are described later.

In a time interval, $t, t+d t$ a number of these labeled atoms per unit volume, $-d A^{*}$, will enter the species $B$. That is, this is the number when there are $A^{*}$ labeled atoms available per unit volume. The number entering per available labeled atom present per unit time is therefore $\left(-d A^{*} / d t\right) / A^{*}$. Moreover, this is the probability per unit time that any given exchangeable atom in an $A$-molecule will enter a $B$-molecule. Now $R$ is the number of $A$-atoms entering positions in the species $B$ per unit volume per unit time, there being $a$ atoms of species $A$ per unit volume available, or $R / a$ is the number of $A$-atoms entering $B$-molecules per available atom per unit time. Therefore, it follows that

$$
\frac{R}{a}=-\frac{1}{A^{*}} \frac{d A^{*}}{d t}
$$

which is the desired relation between $R$ and $A^{*}$.

As an example of the detailed calculation of $-d A^{*} / d t$, and therefore of $R$, the isotopic exchange reaction be- 
tween $\mathrm{B}_{2} \mathrm{H}_{6}$ and $\mathrm{D}_{2}$ will be treated and compared with the experimental data. ${ }^{3}$ The reaction between $\mathrm{B}_{2} \mathrm{H}_{6}$ and $\mathrm{B}_{2} \mathrm{D}_{6}$ has also been studied experimentally. ${ }^{3}$ Its treatment presents some new problems since here there is only one chemical species and its over-all isotopic composition cannot change during the course of the reaction. A method of handling such a case is proposed and the results are compared with the experimental data.

\section{Reaction of $D_{2}$ with $B_{2} H_{6}$}

The system used to study this exchange reaction contained as reactants $\mathrm{B}_{2} \mathrm{H}_{6}$ and $\mathrm{D}_{2}$. As a result of reaction there were presumably produced the following compounds: all possible substituted diboranes, $\mathrm{B}_{2} \mathrm{H}_{5} \mathrm{D}$, $\mathrm{B}_{2} \mathrm{H}_{4} \mathrm{D}_{2} \cdots \mathrm{B}_{2} \mathrm{D}_{6}$, there being some twenty-four of these, all possible substituted borines, $\mathrm{BH}_{3}, \mathrm{BH}_{2} \mathrm{D}, \mathrm{BHD}_{2}$, $\mathrm{BD}_{3}$, and the hydrogens, $\mathrm{HD}$ and $\mathrm{H}_{2}$. The reaction was studied $^{3}$ by essentially analyzing for the total deuterium content present in the hydrogen chemical species after various reaction times. The rate-time data were found to obey the first-order rate law and the first-order rate constant showed a rather peculiar dependence on the initial concentrations.

A possible mechanism for this reaction which will be discussed here involves four basic steps; but, if this mechanism is written in terms of all the distinguishable compounds present (previously enumerated), the number of elementary reactions which contribute to the over-all exchange process is of the order of one hundred. However, as previously mentioned, the first-order rate data can be interpreted, neglecting differences in isotopic reactivity ${ }^{4}$ and making only one further approximation, ${ }^{5}$ by evaluating the constant $R$ which appears in Eq. (1), using the method outlined in the previous section. The basic ideas underlying this approach to the problem can be summarized in the following way. These experimental data were actually obtained in a system having comparable amounts of the different isotopes and also having numerous isotopically different compounds. Then $R$ was determined ${ }^{3}$ from the data with the aid of Eq. (1). Now, $R$, for the case where one isotope is present in trace amounts, has the same value as $R$ for this more complicated case. Therefore, if it is desired to derive a theoretical expression for $R$ in terms

${ }^{3}$ P. C. Maybury and W. S. Koski, J. Chem. Phys. 21, 742 (1953); P. C. Maybury, Dissertation, The Johns Hopkins University, Baltimore, Maryland (1952).

${ }^{4}$ We should like to comment on the neglect of differences in isotopic reactivity in the application of the first-order Eq. (1) to the experimental data. Inasmuch as the data actually obeyed a first-order equation, it may be inferred that the major effect of any difference in isotopic reactivity is on the value of the constant $R$ which appears in Eq. (1). This effect will be considered in an approximate manner in the latter part of this paper.

${ }_{5}$ There is at small times some transient state which is neglected in the application of Eq. (1) to the data. However, the approximation made in neglecting this transient state is justified by the fact that first-order behavior was observed. It may be further remarked that the proposed mechanism is a chain mechanism, and the transient state in gas phase chain reactions would be expected to be established almost immediately. of a detailed reaction mechanism and to compare it with the experimental value, it can be imagined that the reaction was performed under tracer conditions, although the more complex conditions were actually used. This is then done by labeling a small number of atoms and following their progress through the reaction mechanism.

A possible mechanism serving as a basis for this calculation is given by the following set of equations, similar from the viewpoint of chemical species involved to a mechanism previously suggested ${ }^{3}$ :

$$
\begin{gathered}
\mathrm{B}_{2} \mathrm{H}_{6} \underset{k_{-1}}{\stackrel{k_{1}}{\rightleftharpoons}} 2 \mathrm{BH}_{3}, \\
\mathrm{BH}_{3}+\mathrm{HH}^{*} \underset{k_{-2}}{\stackrel{k_{2}}{\rightleftharpoons}} \mathrm{BH}_{3}^{*}+\mathrm{H}_{2}, \\
\mathrm{BH}_{3}^{*}+\mathrm{B}_{2} \mathrm{H}_{6} \stackrel{k_{3}}{\rightarrow} \mathrm{B}_{2} \mathrm{H}_{6}+\mathrm{BH}_{3}, \\
\mathrm{BH}_{3}^{*}+\mathrm{BH}_{3} \stackrel{k_{4}}{\rightarrow} \mathrm{B}_{2} \mathrm{H}_{6} .
\end{gathered}
$$

The labeled atoms are taken to be initially in hydrogen-type molecules so that $A^{*}$ is $\mathrm{H}_{2}{ }^{*}$ here. Let $\left(\mathrm{H}_{2}{ }^{*}\right)$ and $\left(\mathrm{BH}_{3}{ }^{*}\right)$ denote the concentration of $\mathrm{H}_{2}$ and $\mathrm{BH}_{3}$ molecules containing a labeled atom at time $t$. It is emphasized that this labeled atom is not necessarily an isotope but merely a device for the evaluation of the constant $R$. The label vanishes when the atom reaches a position in a $B$-type molecule. Once $R$ is evaluated Eq. (1) may be applied to give the rate of change of the over-all isotopic composition of any chemical species.

Because of the disappearance of the label when the atom enters a position in $B$, i.e., because of the definition of a labeled atom, the reverse of reactions (8) and (9), reforming $\mathrm{BH}_{3}{ }^{*}$, should not be considered in the reaction scheme used to evaluate $R$. Reaction (8) is a reaction whereby the labeled atom of the borine disappears from this species to become part of a diborane molecule.

It follows from this reaction sequence that

$$
\frac{-d \mathrm{H}_{2}{ }^{*}}{d t}=k_{2}\left(\mathrm{BH}_{3}\right)\left(\mathrm{H}_{2}{ }^{*}\right)-k_{-2}\left(\mathrm{BH}_{3}{ }^{*}\right)\left(\mathrm{H}_{2}\right)
$$

where $\left(\mathrm{BH}_{3}\right)$ and $\left(\mathrm{H}_{2}\right)$ denote molar concentrations. Using a steady-state relation for $\mathrm{BH}_{3}{ }^{*}$ we have

$$
\begin{aligned}
\frac{d}{d t}\left(\mathrm{BH}_{3}^{*}\right)=0 & =k_{2}\left(\mathrm{BH}_{3}\right)\left(\mathrm{H}_{2}^{*}\right) \\
& -\left(k_{-2} \mathrm{H}_{2}+k_{3} \mathrm{~B}_{2} \mathrm{H}_{6}+k_{4} \mathrm{BH}_{3}\right)\left(\mathrm{BH}_{3}^{*}\right)
\end{aligned}
$$

Eliminating $\mathrm{BH}_{3}{ }^{*}$ from Eqs. (10) and (11) it follows that

$$
R=-\frac{a}{\left(\mathrm{H}_{2}^{*}\right)} \frac{d\left(\mathrm{H}_{2}^{*}\right)}{d t}=\frac{k_{2} c a}{k_{-2} a / 2\left(k_{4} c+k_{3} b / 6\right)+1}
$$


where we have set $c=\left(\mathrm{BH}_{3}\right), a=2\left(\mathrm{H}_{2}\right)$, and $b=6\left(\mathrm{~B}_{2} \mathrm{H}_{6}\right)$, since $a$ and $b$ denote concentrations in gram atoms per liter while $\left(\mathrm{H}_{2}\right)$ and $\left(\mathrm{B}_{2} \mathrm{H}_{6}\right)$ denote molar concentrations.

The constant $k_{2} / k_{-2}$ is the equilibrium constant of reaction (7). It can be evaluated in terms of the partition functions of the components of this equilibrium. After due cancellation, only a product of symmetry numbers remains, so that

$$
\frac{k_{2}}{k_{-2}}=\frac{\sigma \mathrm{BH}_{3} \sigma \mathrm{H}_{2}}{\sigma \mathrm{BH}_{3}{ }^{*} \sigma \mathrm{H}_{2}}=\frac{6 \times 1}{2 \times 2}=\frac{3}{2} \text {. }
$$

A further simplification of Eq. (12) can be made if the rate of termination of $\mathrm{BH}_{3}{ }^{*}$ chains in this chain process, $k_{4} c\left(\mathrm{BH}_{3}{ }^{*}\right)$, is appreciably less than the rate of propagation, $k_{3}\left(\mathrm{BH}_{3}{ }^{*}\right) b / 6$. This is generally true for chain reactions. A comparison of Eq. (12) with the data leads to the same conclusion here. Thus this equation becomes

$$
R=\frac{k_{2} b^{\frac{1}{2}}\left(k_{1} / 6 k_{-1}\right)^{\frac{1}{2}}}{2 k_{2} / k_{3}+b / a}
$$

where the equilibrium relation, $k_{-1} c^{2}=k_{1} b / 6$, has been introduced.

According to this equation, $R$ is independent of $a$ and proportional to $b^{\frac{1}{2}}$ when $a$ is relatively high such that $2 k_{2} / k_{3} \gg b / a$. When $a$ is relatively low, i.e., when $2 k_{2} / k_{3} \ll b / a$, then $R$ is proportional to $a$ and also to $b^{\frac{1}{3}}$. This behavior was observed experimentally ${ }^{6}$ with the exception of the square-root dependence on the diborane concentration at low $a$ which has not yet been tested. It would be interesting to restudy experimentally this aspect of the problem.

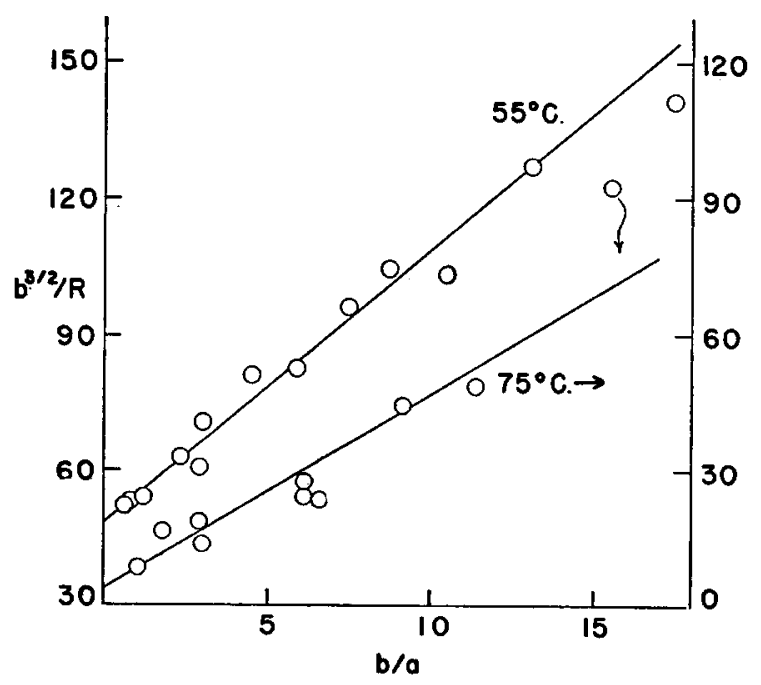

FIG. 1. Dependence of $b y / R$ on the ratio of the diborane to deuterium concentration, $b / a$, for $55^{\circ} \mathrm{C}$ (left-hand ordinate) and $75^{\circ} \mathrm{C}$ (right-hand ordinate).

see reference 3 . It is observed that our $a, \mathbf{H}_{2}$, is denoted by $b$ there, and conversely.
According to Eq. (14) a plot of $b^{\frac{3}{2}} / R$ vs $b / a$ should be a straight line whose ratio of slope to intercept is $k_{3} / 2 k_{2}$. Within experimental error this plot is found to be linear as seen from Fig. 1 . At $55^{\circ} \mathrm{C}, k_{3} / k_{2}$ is estimated in this manner to be 0.25 . At $75^{\circ} \mathrm{C}$ this ratio is definitely higher, but its exact value as inferred from Fig. 1 is seen to be uncertain. It would appear that the reaction of a borine with diborane has a somewhat higher activation energy than that of borine with hydrogen. This is rather unexpected. When $R$ is independent of $a, R=k_{3}\left(k_{1} b^{3} / 6 k_{-1}\right)^{\frac{1}{2}} / 2$, and the activation energy $E_{\text {act }}$ for the over-all reaction becomes

$$
E_{\text {act }}=E_{3}+\frac{\Delta \mathrm{H}_{1}}{2}
$$

where $\Delta \mathrm{H}_{1}$ is the heat of reaction of (6). Furthermore, $E_{\text {act }}$ equals $20.4 \pm 2 \mathrm{kcal} \mathrm{mole}^{-1}$.

The foregoing analysis shows that the peculiar dependence of $R$ on $a$ and $b$ can be consistent with a homogeneous mechanism. It had earlier been assumed ${ }^{3}$ that this behavior was evidence for heterogeneous reaction. Actually the reaction is at least slightly heterogeneous, the rate increasing somewhat less than twofold at $55^{\circ} \mathrm{C}$ when the surface was increased tenfold, while at $75^{\circ} \mathrm{C}$ the rate was essentially unchanged. Such a slight surface effect will not affect the general agreement obtained above. Naturally, this does not rule out any heterogeneous mechanism consistent with these experimental observations, but there does not appear to be any need for invoking it at the present time.

It is interesting to note that the reaction rate was appreciably reduced at $55^{\circ} \mathrm{C}$ and at $75^{\circ} \mathrm{C}$, five- or sixfold in some cases, by the addition of $\mathrm{CO}_{2}$ or $\mathrm{N}_{2}$ though not of He. While this might, as previously suggested, ${ }^{3}$ be evidence for a surface reaction, an interesting alternative might be that the unsaturated borine is, possibly, partially associated with such molecules, thus reducing its reactivity. The effect of added gases on this and on other, equally slow, reactions of diborane is therefore of considerable interest.

\section{Reaction of $B_{2} D_{6}$ with $B_{2} H_{6}$}

In the exchange reaction between $\mathrm{B}_{2} \mathrm{H}_{6}$ and $\mathrm{B}_{2} \mathrm{D}_{6}$, the percentage deuterium in a given chemical species cannot change, there being but one such species, diborane. Thus the arguments of the previous sections cannot be applied to the treatment of data on this reaction without some further modification, and the modified treatment of these results presented here will be somewhat less rigorous.

Presumably many isotopic isomers of diborane are formed during the reaction, ranging from $\mathrm{B}_{2} \mathrm{H}_{6}$, $\mathrm{B}_{2} \mathrm{H}_{6} \mathrm{D} \cdots$ to $\mathrm{B}_{2} \mathrm{D}_{6}$. The rate of disappearance of $\mathrm{B}_{2} \mathrm{H}_{6}$ in mixtures initially equimolar with respect to the two reactants, $\mathrm{B}_{2} \mathrm{H}_{6}$ and $\mathrm{B}_{2} \mathrm{D}_{6}$, was followed ${ }^{3}$ by measuring the decrease in intensity of the infrared absorption band 
at $974 \mathrm{~cm}^{-1}$, associated with a vibration of two $\mathrm{BH}_{2}$ groups with respect to each other. In this vibrational mode the terminal hydrogens move and the bridge hydrogens remain stationary. The intensity of this absorption band should decrease when any of the four terminal hydrogens changes positions with a deuterated atom. Experimentally the rate of decrease of intensity of this band obeys, at least approximately, a first-order relation.

For purposes of interpretation of these data the group of four terminal positions of the hydrogens in diborane is regarded as forming one species and the two bridge positions, another. It follows that the rate of change of deuterium content in either of these species would obey the first-order Eq. (1), neglecting differences in isotopic reactivity, where $a$ and $b$ denote the concentrations of the terminal and bridge species in gram atoms per liter. Inasmuch as there are twice as many terminal atoms as bridge atoms, $a=2 b$ and this equation becomes

$$
R t=-\frac{a}{3} \ln (1-F)
$$

where $R$, defined in a previous section, is the rate per unit volume at which atoms of one species assume positions in the other species.

Now one isotopic isomer of the terminal species is that member containing hydrogens in all four terminal positions. It does not necessarily follow that this member would obey the first-order relation Eq. (16), where $(1-F)$ would now be the ratio $\left(p_{t}-p_{\infty}\right)$ / $\left(p_{0}-p_{\infty}\right)$, the $p$ 's denoting partial pressure of this constituent at the designated times $0, t$, and $\infty$. However, it seems reasonable to suppose that this would approximately be the case and this will constitute the major assumption of this section.

In order to calculate $R$, we label a very small number of atoms such that

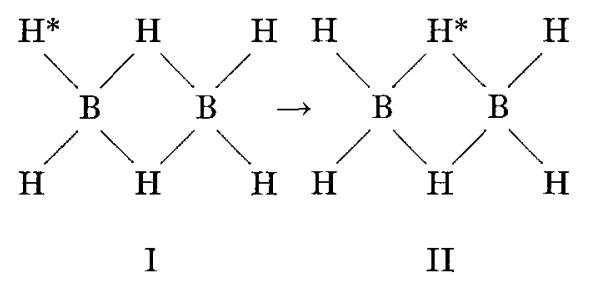

Each labeled atom is initially in a terminal position, as in $I$, say, no molecule having more than one labeled atom. The rate at which these atoms enter a bridge position $I I$ is then estimated. As before, the subsequent fate of any labeled atom once it has entered the other species (here the bridge positions) is not relevant to the calculation of $R$ and for this reason the reverse of certain steps in the following mechanism is not considered in the calculation. The label in the species $I I$ is preserved only to indicate that the labeled atom entered a diborane molecule in the bridge position. A possible mechanism for the self-deuteration, similar structurally to that suggested by Maybury and Koski ${ }^{3}$ is as follows:

$$
\begin{aligned}
& \mathrm{B}_{2} \mathrm{H}_{6} \underset{k_{-1}}{\stackrel{k_{1}}{\rightleftharpoons}} 2 \mathrm{BH}_{3}, \\
& I \underset{k_{-1}}{\stackrel{k_{1}}{\rightleftharpoons}} \mathrm{BH}_{3}{ }^{*}+\mathrm{BH}_{3}, \\
& \mathrm{BH}_{3}{ }^{*}+\mathrm{BH}_{3} \stackrel{k_{-1} \prime}{\rightarrow} I I, \\
& \mathrm{BH}_{3}{ }^{*}+\mathrm{B}_{2} \mathrm{H}_{6} \stackrel{k_{3}{ }^{\prime}}{\rightarrow} \mathrm{BH}_{3}+I I, \\
& \mathrm{BH}_{3}{ }^{*}+\mathrm{B}_{2} \mathrm{H}_{6} \underset{k_{3}{ }^{\prime \prime \prime}}{\stackrel{k_{3}{ }^{\prime \prime}}{\rightleftharpoons}} \mathrm{BH}_{3}+I, \\
& \mathrm{BH}_{3}+I \stackrel{k_{3}{ }^{\mathrm{IV}}}{\rightarrow} \mathrm{BH}_{3}+I I .
\end{aligned}
$$

We let $I$ denote the concentration of labeled atoms present in form $I$ at any time $t ; I$ decreases with time from a value equal to the initial number of labeled atoms at $t=0$ to zero at $t=\infty$.

According to the aforementioned mechanism we have

$$
-\frac{d I}{d t}=\frac{d I I}{d t}=\left(k_{-1}{ }^{\prime \prime} c+k_{3}{ }^{\prime} \mathrm{B}_{2} \mathrm{H}_{6}\right)\left(\mathrm{BH}_{3}{ }^{*}\right)+k_{3}{ }^{\mathrm{Iv}} c I \text {. }
$$

A steady-state equation for $\left(\mathrm{BH}_{3}{ }^{*}\right)$ is

$$
\begin{aligned}
\frac{d\left(\mathrm{BH}_{3}{ }^{*}\right)}{d t}=0 & =\left(k_{1}+{k_{3}}^{\prime \prime \prime} c\right) I-\left(\mathrm{BH}_{3}{ }^{*}\right) \\
& \times\left[\left(k_{-1}{ }^{\prime}+k_{-1}{ }^{\prime \prime}\right) c+\left(k_{3}{ }^{\prime}+{k_{3}}^{\prime \prime}\right)\left(\mathrm{B}_{2} \mathrm{H}_{6}\right)\right] .
\end{aligned}
$$

Introducing this equation for $\left(\mathrm{BH}_{3}{ }^{*}\right)$ into Eq. (23) we have

$$
-\frac{1}{I} \frac{d(I)}{d t}=\frac{\left(k_{-1}{ }^{\prime \prime} c+k_{3}{ }^{\prime} \mathrm{B}_{2} \mathrm{H}_{6}\right)\left(k_{1}+k_{3}{ }^{\prime \prime \prime} c\right)}{\left(k_{-1}{ }^{\prime}+k_{-1}{ }^{\prime \prime}\right) c+\left(k_{3}{ }^{\prime}+k_{3}{ }^{\prime \prime}\right) \mathrm{B}_{2} \mathrm{H}_{6}}+k_{3}{ }^{\mathrm{Iv}} c .
$$

Now, the $k_{-1}$ 's differ only by a small numerical factor at most, as do the $k_{3}$ 's. As in the previous section, we let $k_{-1} c \ll k_{3} \mathrm{~B}_{2} \mathrm{H}_{6}$ and since $k_{1} \mathrm{~B}_{2} \mathrm{H}_{6}=k_{-1} c^{2}$ it follows also that $k_{1} \ll k_{3} c$. Thus Eq. (25) becomes

$$
-\frac{1}{I} \frac{d I}{d t}=\left(\frac{k_{3}{ }^{\prime} k_{3}{ }^{\prime \prime \prime}}{k_{3}{ }^{\prime}+k_{3}{ }^{\prime \prime}}+k_{3}{ }^{\mathrm{IV}}\right) c .
$$

Inasmuch as all $k_{3}$ 's are approximately equal the righthand side is about $k_{3} c$. A somewhat more accurate figure is ${ }^{7} k_{3} c / 6$. The total rate of exchange $R$ of the

\footnotetext{
7 The relative values of the various $k_{3}$ 's can be calculated as follows. It is emphasized that symmetry effects, not isotopic effects, are being estimated in this footnote. The ratio $k_{3}{ }^{\prime \prime} / k_{3}{ }^{\prime \prime}$ is the equilibrium constant of (21) and is estimated from the symmetry numbers of the components to be $4 / 3$. Also $k_{3}{ }^{\prime \prime}=2 k_{3}{ }^{\prime}$ since there are twice as many equivalent collision complexes, resulting from a collision of a $\mathrm{BH}_{3}{ }^{*}$ and $\mathbf{a ~}_{2} \mathrm{H}_{6}$, which lead to $I$ as compared
} 
terminal atoms per unit volume is $(-a / I)(d I / d t)$ since $(-1 / I) d I / d t$ is the rate of exchange for a given terminal atom and since there are $a$ terminal atoms per unit volume. Combining this with Eqs. (16) and (26) we obtain a first-order rate constant, $k$, for the rate of disappearance of the $974 \mathrm{~cm}^{-1}$ band.

$$
k=\frac{1}{t} \ln (1-F)=k_{3} c / 2=k_{3}\left(k_{1} / k_{-1}\right)^{\frac{1}{2}}\left(\mathrm{~B}_{2} \mathrm{H}_{6}\right)^{\frac{1}{2}} / 2
$$

where $\left(\mathrm{B}_{2} \mathrm{H}_{6}\right)$ is the sum of concentrations of all diborane type molecules, in gram moles per liter. The square root dependence of Eq. (27) has been verified experimentally. ${ }^{3}$ According to Eq. (27), the activation energy of this reaction is $\left(E_{3}+\Delta H_{1} / 2\right)$, the same as that for the deuteration of diborane at relatively high pressures of deuterium, if isotopic effects be neglected. This too has been verified within experimental error, the activation energy for the self-deuteration reaction being $21.8 \pm 3 \mathrm{kcal} \mathrm{mole}^{-1}$. However the absolute rate of the self-deuteration reaction is substantially faster than the deuteration reaction, the value of $k_{3}\left(k_{1} / k_{-1}\right)^{\frac{1}{2}}$ at $44^{\circ} \mathrm{C}$ inferred ${ }^{8}$ from the former reaction being about three hundred fold greater than that estimated from the latter process. No effect of added Pyrex surface or of inert gases was found for the self-deuteration reaction, perhaps because it is so rapid that a much larger variation of these factors would be needed to obtain an effect comparable to that observed for the deuteration reaction. It is of interest to speculate as to the reason for this relative rapidity of the selfdeuteration reaction. First, the mechanisms discussed

with those leading to II. Further, the sum of these two constants $\left(k_{3}{ }^{\prime}+k_{3}{ }^{\prime \prime}\right)$ must equal $k_{3}$ since an effective collision must produce $I$ or $I I$, and $k_{3}$, the rate constant for $(8)$, is actually equal to the number of effective collisions. In this manner it is concluded that $3 k_{3}{ }^{\prime}=3 k_{3}{ }^{\prime \prime} / 2=2 k_{3}{ }^{\prime \prime \prime}=k_{3}$. The value of $k_{3} \mathrm{IV}^{\prime} / k_{3}$, depends on the exact nature of the collision complex and can be shown to lie between 0 and $\frac{1}{4}$. It will be ignored in our approximate treatment, the error being at most a factor of 2.5 .

${ }^{8}$ In the application of the data to Eq. (14) it was seen that in the lower $b / a$ range at $55^{\circ} \mathrm{C}$ the rate of the deuteration reaction was a direct measure of $k_{3}\left(k_{1} / k_{-1}\right)$, so that the latter could be inferred at once from the reaction rate. From the comparison of the data obtained at $55^{\circ} \mathrm{C}$ and $75^{\circ} \mathrm{C}$ and given earlier, it appears that this approximation (which amounts to neglecting, in Eq. (14), $b / a$ in comparison with $2 k_{2} / k_{3}$ ) becomes increasingly valid at lower temperatures, $45^{\circ} \mathrm{C}$ for example. The value of $b / a$ used at $45^{\circ} \mathrm{C}$ was about 2 , while $2 k_{2} / k_{3}$ should at least equal 8 , the value at $55^{\circ} \mathrm{C}$ estimated previously. That is, at $45^{\circ} \mathrm{C}$ the value of $k_{3}\left(k_{1} / k_{-1}\right)$ could be inferred directly from the observed reaction rate.

It may be remarked that the large difference in rates of selfdeuteration and deuteration reactions will prevail at other adjacent temperatures, for the temperature coefficients of both reactions were seen to be essentially the same. may be incorrect, but, inasmuch as they make several predictions, they can be further tested, at least partially. Second, if the assumption mentioned in reference 7 were in error, this factor could be reduced to $300 / 2.5$, a still rather high value. Again, the reaction vessel of these two systems differed, the self-deuteration reaction having been studied in an infrared absorption cell with its salt windows, and with its walls of unspecified composition. It is also possible that in the deuteration reaction the rate of change of deuterium content of either of the reactants is slower than the rate of disappearance of the $974 \mathrm{~cm}^{-1}$ absorption band. It would be of interest to re-examine this reaction using both methods of analysis simultaneously. Another possible reason lies in isotopic effects. ${ }^{4}$ If $\mathrm{B}_{2} \mathrm{D}_{6}$ dissociates more readily than $\mathrm{B}_{2} \mathrm{H}_{6}$ into borines then the self-deuteration reaction will presumably proceed at a lower temperature, depending upon the isotopic effect of $k_{3}$. The over all activation energy would almost undoubtedly also be lower but could equal the other activation energy within the experimental error. At the prevailing temperatures, a difference in reaction rate of a factor of one hundred can be equivalent to a difference in activation energy of $3 \mathrm{kcal} \mathrm{mole}{ }^{-1}$. On the basis of a recent assignment of infrared bands ${ }^{9}$ we have estimated the relative equilibrium constants for the dissociation of $\mathrm{B}_{2} \mathrm{H}_{6}$ and of $\mathrm{B}_{2} \mathrm{D}_{6}$ into $\mathrm{BH}_{3}$ and $\mathrm{DB}_{3}$, respectively. If one assumes the $\mathrm{BH}$-stretching and bending frequencies in borine to be the average values observed for the terminal hydrogens in diborane, (for $\mathrm{B}_{2} \mathrm{H}_{6}, 2563 \mathrm{~cm}^{-1}$ and $1179 \mathrm{~cm}^{-1}$ respectively, and for $\mathrm{B}_{2} \mathrm{D}_{6}, 1921 \mathrm{~cm}^{-1}$ and $905 \mathrm{~cm}^{-1}$ ) then the ratio of the equilibrium constants is about unity. However, this assumption is open to question since the terminal $\mathrm{BH}$-bonds in $\mathrm{B}_{2} \mathrm{H}_{6}$ are presumably approximately tetrahedral, while the $\mathrm{BH}$-bonds are probably trigonal in $\mathrm{BH}_{3}$. If the bonds in borine are actually stiffer than those in diborane, then $\mathrm{B}_{2} \mathrm{H}_{6}$ is more stable than $\mathrm{B}_{2} \mathrm{D}_{6}$ with respect to dissociation into borines. In this connection it is interesting to observe that the $\mathrm{CH}$-stretching frequencies in ethane, which has tetrahedral $\mathrm{CH}$-bonds, are greater than those in ethylene, which has trigonal bonds. The $\mathrm{CH}$ bending frequencies are, on the other hand, in the reverse order. It would be of interest to see if the rate of reaction of $\mathrm{B}_{2} \mathrm{D}_{6}$ with $\mathrm{H}_{2}$ proceeds at a lower temperature than the reaction of $\mathrm{B}_{2} \mathrm{H}_{6}$ with $\mathrm{D}_{2}$. A comparative study of some of the other reactions of $\mathbf{B}_{2} \mathrm{H}_{6}$ and $\mathrm{B}_{2} \mathrm{D}_{6}$ whose mechanisms appear to involve a preliminary dissociation into borines is also relevant.

\footnotetext{
${ }^{9}$ R. C. Lord and E. Nielsen, J. Chem. Phys. 19, 1 (1951).
} 